\title{
Matériáta
}

ISSN 1517-7076

Revista Matéria, v. 13, n. 2, pp. 365 - 373, 2008

http://www.materia.coppe.ufrj.br/sarra/artigos/artigo11000

\section{Caracterización de tres aleaciones superplásticas utilizando análisis mecánico dinámico (DMA)}

\author{
MARTÍNEZ-FLORES, E. E. II ; NEGRETE, J. II; TORRES-VILLASEÑOR, G. " \\ I Facultad de Ingeniería-UASLP, Dr. Manuel Nava 8, Zona Universitaria, 78290 San Luis Potosí, S. L. P., \\ México. \\ e-mail: emartine@uaslp.mx

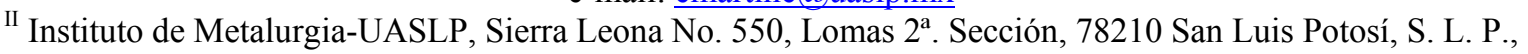 \\ México. \\ e-mail: jnegrete@uaslp.mx \\ III Instituto de Investigaciones en Materiales-UNAM, Apdo. Postal 70-360, 04519 México, D. F. México. \\ e-mail: gtorres@servidor.unam.mx
}

\section{RESUMEN}

El Análisis Mecánico Dinámico (DMA) es un método que tradicionalmente se utiliza para el análisis de polímeros. Está técnica es muy sensible a cambios en las propiedades de los materiales, como la transición vítrea en polímeros. Se puede utilizar para evaluar el ángulo de desfasamiento (tan $\boldsymbol{\delta}$ ), el módulo de almacenamiento $\left(\boldsymbol{E}^{\prime}\right)$ el módulo de pérdida $\left(\boldsymbol{E}^{\prime}\right)$ y recientemente las temperaturas de transformación en aleaciones metálicas.

Se utilizó DMA para estudiar tres diferentes aleaciones: $\mathrm{Zn}-21 \% \mathrm{Al}-2 \% \mathrm{Cu}$; Cd-17\% $\mathrm{Zn}$ y $\mathrm{Sn}-38 \% \mathrm{~Pb}$, cada una en condición superplástica y no superplástica. Los resultados mostraron la presencia de un pico en la curva de módulo de pérdida el cual se puede relacionar con la temperatura superplástica para cada una de las aleaciones analizadas. Es posible que el incremento en el módulo de pérdida se relacione con procesos de reacomodamiento de bordes de grano desde un estado de no-deslizamiento hacia un estado de deslizamiento. La activación de un gran número de bordes de grano puede relacionarse con la disminución en el esfuerzo cortante requerido para vencer la energía de cohesión entre granos cuando el material se encuentra en la temperatura superplástica.

Palabras clave: Análisis Mecánico Dinámico, superplasticidad, temperatura homóloga.

\section{Characterization of three superplastic alloys using dynamic mechanic analysis}

\section{ABSTRACT}

Dynamic Mechanical Analysis (DMA) is traditionally a standard method in polymer analysis. This technique is very sensitive to changes in materials properties, like glass transition in polymers. It can be used to measure phase difference ( $\tan \boldsymbol{\delta})$, storage modulus $\left(\boldsymbol{E}^{\prime}\right)$, loss modulus $\left(\boldsymbol{E}^{\prime}\right)$ and recently transformation temperatures for metal alloys.

Three different alloys: $\mathrm{Zn}-21 \% \mathrm{Al}-2 \% \mathrm{Cu}, \mathrm{Cd}-17 \% \mathrm{Zn}$ and $\mathrm{Sn}-38 \% \mathrm{~Pb}$, each one in a superplastic and non-superplastic condition have been studied using Dynamic Mechanical Analysis (DMA). Results showed a clear peak of the loss modulus which is related to the superplastic temperature for each analyzed alloy. It is possible that the increase of the loss modulus is related with the accommodation of grain boundaries from non-sliding state to sliding state. The activation of a higher number of grain boundaries can be connected with a lower shear stress required to overcome the cohesion energy between grains at the superplastic temperature.

Keywords: Dynamic Mechanical Analysis, superplasticity, homologous temperature.

\section{$1 \quad$ INTRODUCCIÓN}

Los primeros intentos de experimentos oscilatorios para medir la elasticidad de un material se hicieron a principios del siglo pasado [1], fue a partir de la década de los 60's cuando el soporte teórico 
disponible permitió desarrollar equipos comercialmente accesibles. Sin embargo, hasta la década de los 80's el costo y la sensibilidad de los equipos de instrumentación permitieron que esta técnica fuese rápida y fácil de usar. El análisis mecánico dinámico (DMA) es una técnica que se ha utilizado ampliamente en la caracterización de polímeros debido a que proporciona información, tanto para transiciones primarias como para transiciones de segundo y tercer orden que no se pueden identificar con facilidad con otras técnicas $[\underline{1}$, 2]. En la actualidad esta técnica ofrece alternativas para evaluar propiedades viscoelásticas y de amortiguamiento en sólidos. Recientemente en análisis mecánico dinámico se ha utilizado para el estudio de metales y aleaciones; en estos trabajos se reportan las propiedades de amortiguamiento de las aleaciones, o bien se estudian las transformaciones de fase que ocurren en aleaciones con memoria de forma [ $\underline{3}-\underline{7}]$.

En este trabajo se estudiará la variación de el módulo de pérdida (E”), el módulo de almacenamiento $\left(\boldsymbol{E}^{\prime}\right)$ y la $\tan \boldsymbol{\delta}$, en función de la temperatura y se buscará, si existe, alguna relación con el fenómeno superplástico en metales. Se utilizará la técnica de Análisis Mecánico Dinámico sobre tres aleaciones con reconocido comportamiento superplástico [묘 $\underline{10}$ ]. Las aleaciones elegidas son $\mathrm{SnPb}$ y $\mathrm{CdZn}$ en la composición eutéctica y $\mathrm{ZnAlCu}$ en la composición eutectoide, todas estas aleaciones presentan superplasticidad a temperaturas inferiores a $\operatorname{los} 350^{\circ} \mathrm{C}$ lo cual está dentro del intervalo de trabajo del equipo de Análisis Mecánico Dinámico disponible.

\section{MATERIALES Y MÉTODOS}

Durante la prueba de DMA, una placa de metal se somete a una ligera deformación debida a la aplicación de una fuerza oscilatoria sobre la probeta, lo que permite estudiar la presencia de efectos viscosos y elásticos en el material. A partir de la respuesta del material a la onda de esfuerzo se calcula, un módulo complejo (E*). El módulo complejo es una medida de la resistencia que opone el material a la deformación y reúne ambas respuestas: elástica, a través del módulo de almacenamiento $(\boldsymbol{E})$, y viscosa a través del módulo de pérdida $(\boldsymbol{E}$ ”), estos valores se monitorean como función de la frecuencia y la temperatura.

$$
E^{*}(\omega, T)=E^{\prime}(\omega, T)+i E^{\prime \prime}(\omega, T)
$$

El módulo de almacenamiento $\left(\boldsymbol{E}^{\prime}\right)$ refleja la respuesta elástica del material. El módulo de pérdida $\left(\boldsymbol{E}^{\prime}\right)$ describe la energía por deformación que se disipa o se pierde por fricción y movimientos internos producidos por movilidad entre granos, por lo que este valor representa la capacidad del material para reorientar o modificar su microestructura. La razón entre estos efectos se conoce como tan $\delta$, fricción interna o amortiguamiento.

$$
\tan \delta=\frac{E^{\prime \prime}}{E^{\prime}}
$$

Los experimentos de análisis mecánico dinámico se efectuaron en un equipo TA Q800 usando una pinza para prueba en voladizo sencillo (single cantilever clamp). Se utilizaron las aleaciones $\mathrm{Zn}-21 \% \mathrm{Al}$ $2 \% \mathrm{Cu}(\mathrm{ZnAlCu})$; Cd-17\%Zn (CdZn) y $\mathrm{Sn}-38 \% \mathrm{~Pb}$ ( $\mathbf{S n P b})$, en todos los casos se considera \% en masa. Las probetas de prueba fueron placas de aproximadamente $4 \mathrm{~mm}$ x $1 \mathrm{~mm}$ x $35 \mathrm{~mm}$ de las aleaciones en estado de colada (no superplástico) y laminadas (superplástico). Todas las pruebas se efectuaron utilizando una amplitud de oscilación de $15 \mu \mathrm{m}$ y una velocidad de calentamiento de $3^{\circ} \mathrm{C} / \mathrm{min}$. Los resultados se analizaron usando el software TA Universal Análisis 2000.

El equipo de DMA trabaja en un intervalo de frecuencias de 0.01 a $200 \mathrm{~Hz}$. En la primera etapa de este trabajo se estudió la variación de $\boldsymbol{E}, \boldsymbol{E}^{\prime}$ ' y $\tan \boldsymbol{\delta}$ en función de la frecuencia y la temperatura para la aleación $\mathrm{ZnAlCu}$, las frecuencias utilizadas están entre 0.05 y $100 \mathrm{~Hz}$ con barrido de temperatura de 25 a $300^{\circ} \mathrm{C}$. Con estos barridos intentamos conocer las frecuencias a las cuales aparecen singularidades en los valores de $E^{\prime}, E^{\prime}$ y $\tan \delta$.

En la segunda etapa se analizó el comportamiento de las tres diferentes aleaciones en función de la temperatura manteniendo constante la frecuencia de trabajo de acuerdo con los resultados obtenidos en la primera etapa. El intervalo de temperatura para la aleación ZnAlCu fue 25 a $300^{\circ} \mathrm{C}$; para la aleación CdZn se hizo un barrido de 25 a $200^{\circ} \mathrm{C}$ y para $\mathrm{SnPb}$ de 25 a $140^{\circ} \mathrm{C}$. 


\section{RESULTADOS}

En la etapa preliminar de este trabajo se analizaron los cambios de E', E" y $\tan \delta$ en función de la frecuencia y la temperatura usando la aleación $\mathrm{Zn}-21 \% \mathrm{Al}-2 \% \mathrm{Cu}$, tanto en estado de colada, como con microestructura de granos finos. Este primer experimento determinará las condiciones de trabajo más adecuadas para comparar el comportamiento de las aleaciones en estudio.

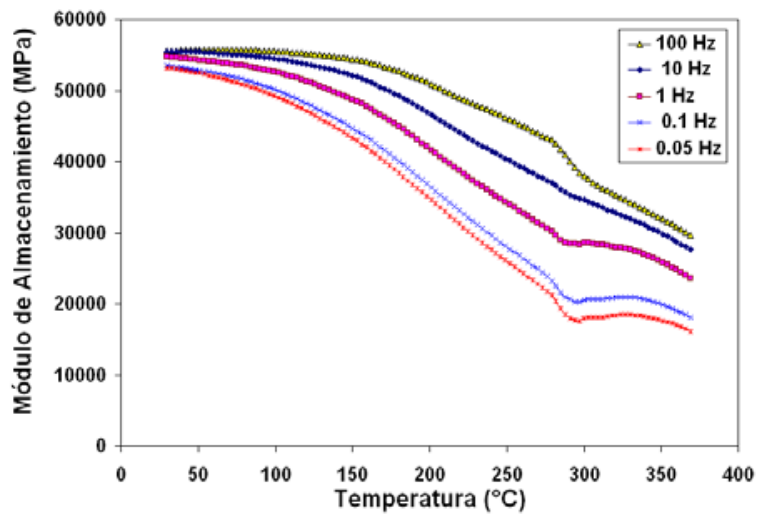

(a)

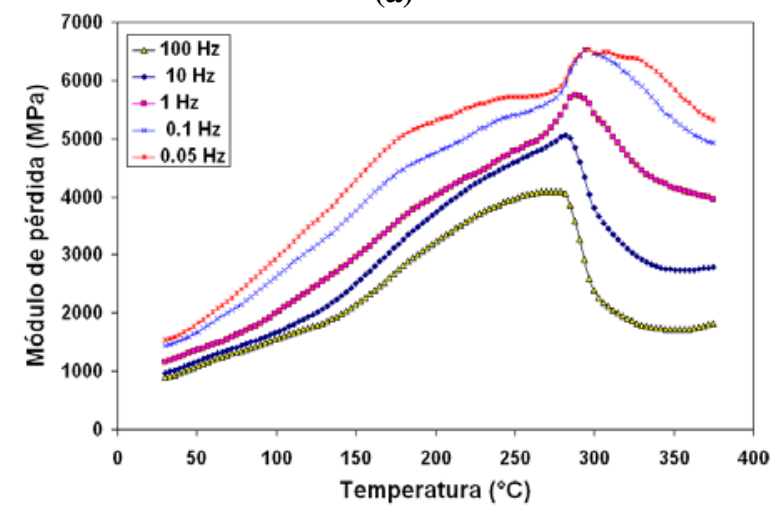

(b)

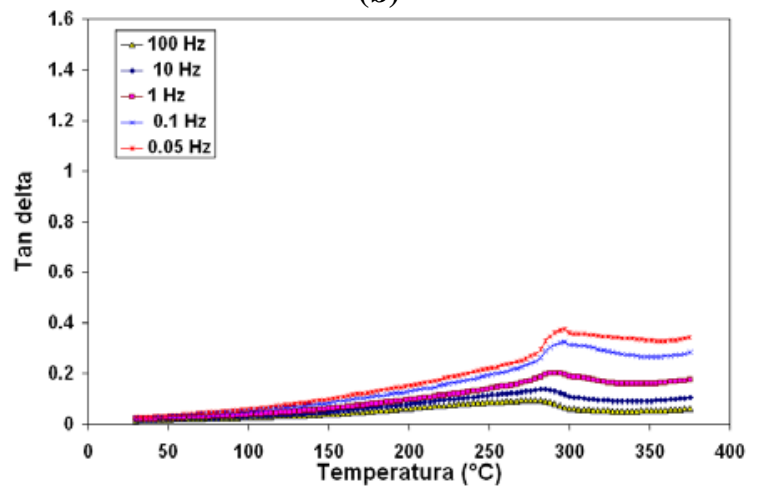

(c)

Figura 1: Variación del módulo de almacenamiento (a),el módulo de pérdida (b) y $\tan \delta$ (c) en función de la frecuencia y la temperatura para la aleación $\mathrm{Zn}-21 \% \mathrm{Al}-2 \% \mathrm{Cu}$ en estado de colada.

Los resultados E', E" y $\tan \delta$ usando frecuencias de $0.05,0.1,1,10$ y $100 \mathrm{~Hz}$ y un barrido de temperatura entre 25 y $350^{\circ} \mathrm{C}$ para la aleación $\mathrm{Zn}-21 \% \mathrm{Al}-2 \% \mathrm{Cu}$ en estado de colada se presentan en la Figura 1(a, b, c). El módulo de almacenamiento, Figura 1(a), que está relacionado con el módulo elástico, disminuye con la temperatura para todas las frecuencias utilizadas. Esta disminución es mucho más pronunciada para $0.05 \mathrm{~Hz}$ y menos marcada para $100 \mathrm{~Hz}$. Se observa un pequeño pico entre 279 y $300^{\circ} \mathrm{C}$, éste se puede asociar con la transformación eutectoide de esta aleación, la cual es una aleación binaria que se ha modificado con $2 \%$ de cobre. La temperatura eutectoide reportada para el sistema binario $\mathrm{Zn}$-Al que es de $277^{\circ} \mathrm{C}[\underline{11}]$. 
La $\tan \delta$, Fig. 1(c), toma valores del orden de 0.01 en el inicio de la prueba para todas las frecuencias y alcanza un valor máximo de aproximadamente 0.4 para una frecuencia de $0.05 \mathrm{~Hz}$ y $296^{\circ} \mathrm{C}$. Los valores reportados de $\tan \delta$ para metales y aleaciones no superplásticas son del orden de 10-3 o menores [12]. Nuestros resultados indican que esta aleación presenta una capacidad de amortiguamiento grande y que esta capacidad aumenta con la temperatura para todas las frecuencias utilizadas. El aumento en los valores de amortiguamiento es más importante para una frecuencia de $0.05 \mathrm{~Hz}$, lo que indica la presencia de un mecanismo de absorción de energía que actúa a bajas frecuencias el cuál provoca una gran respuesta viscoelástica del material. Es importante considerar que las pruebas efectuadas usando una frecuencia de 0.05 $\mathrm{Hz}$ tardan 24 horas o más, por lo que no es recomendable utilizar frecuencias tan bajas. Podemos observar en las curvas de E', E" y tan $\delta$ de la Figura 1 que para una frecuencia de $1 \mathrm{~Hz}$, la respuesta viscoelástica ya es importante, con la ventaja de que el tiempo de prueba se reduce a solo un par de horas.

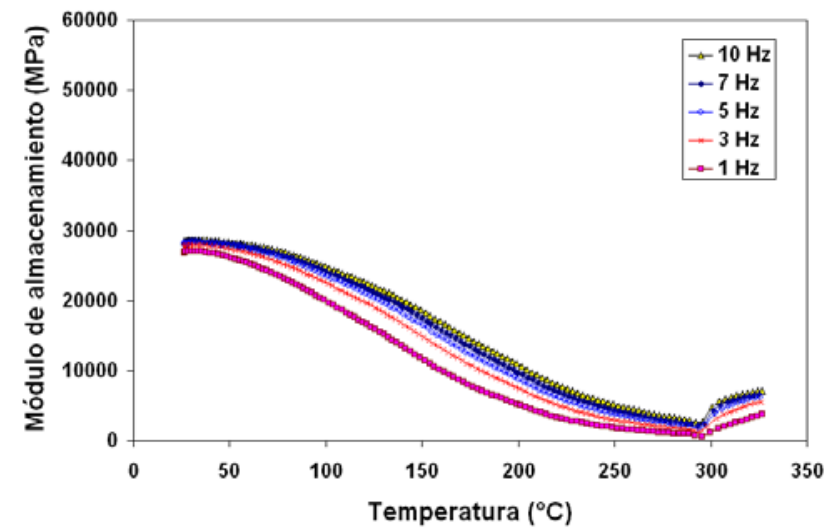

(a)

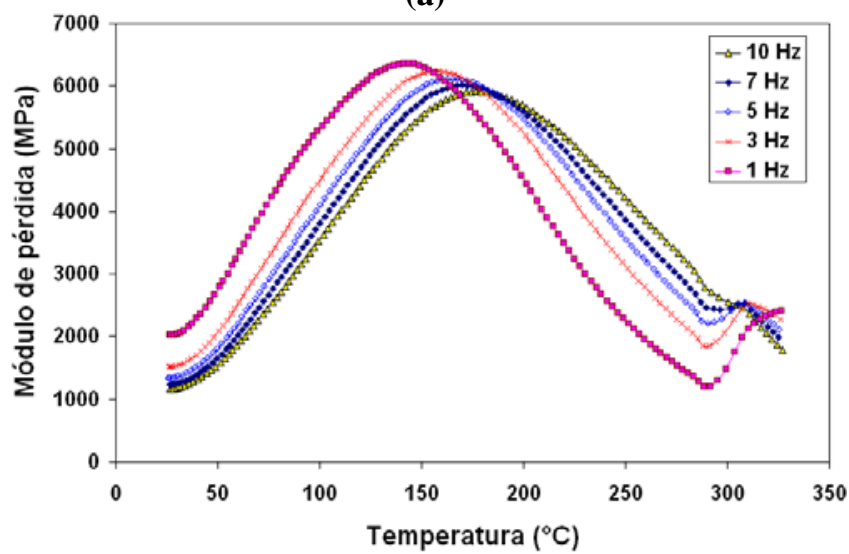

(b)

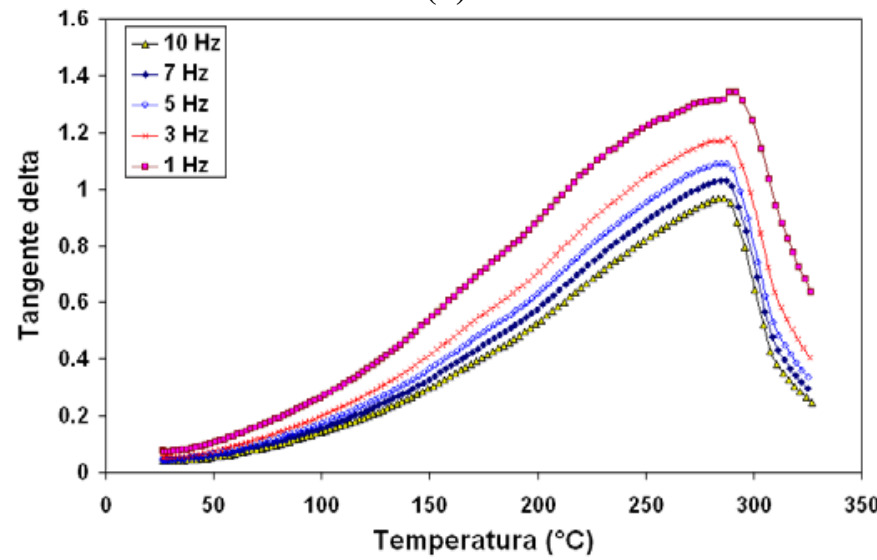

(c)

Figura 2: Variación del módulo de almacenamiento (a), el módulo de pérdida (b) y tan $\delta$ (c) en función de la frecuencia y la temperatura para la aleación $\mathrm{Zn}-21 \% \mathrm{Al}-2 \% \mathrm{Cu}$ laminada. 


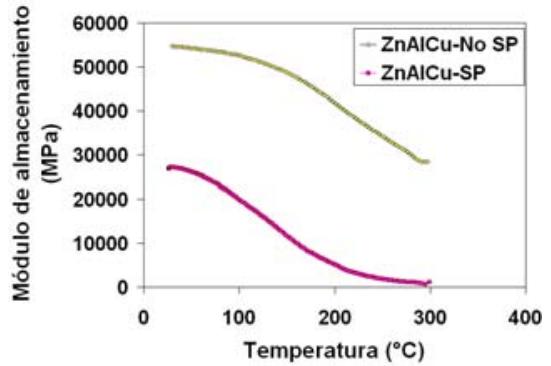

(a)

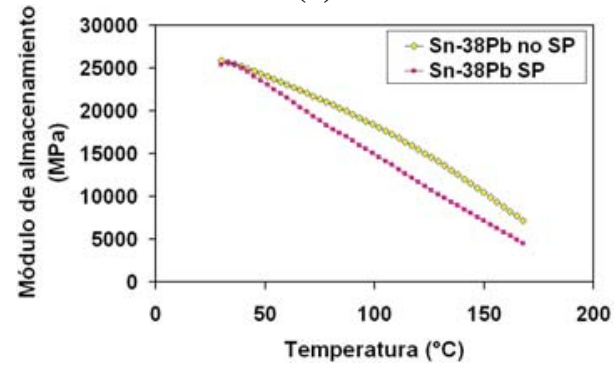

(c)

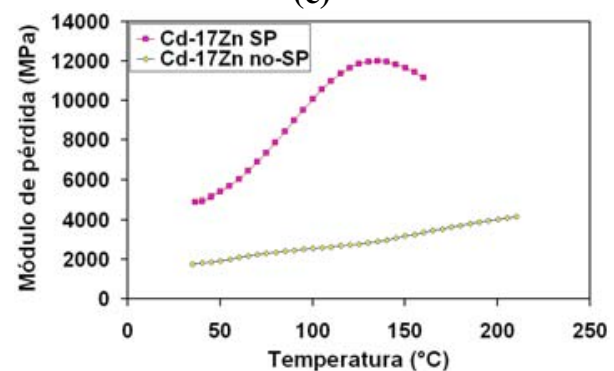

(e)

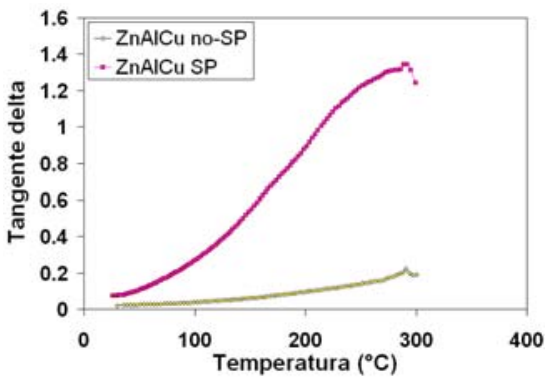

(g)

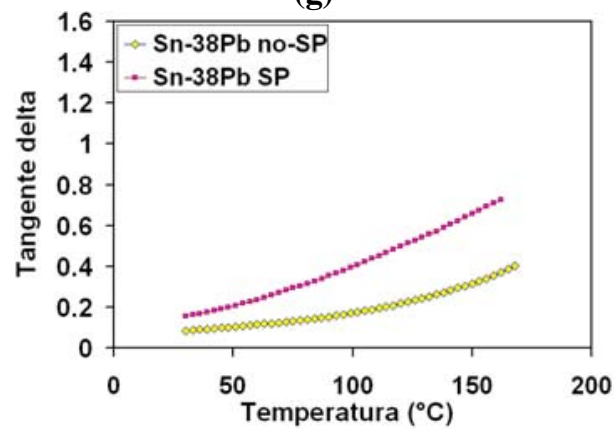

(i)

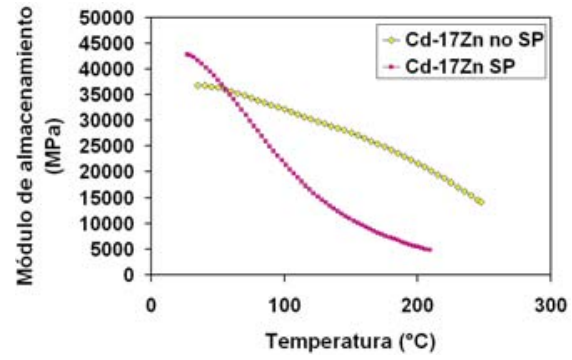

(b)

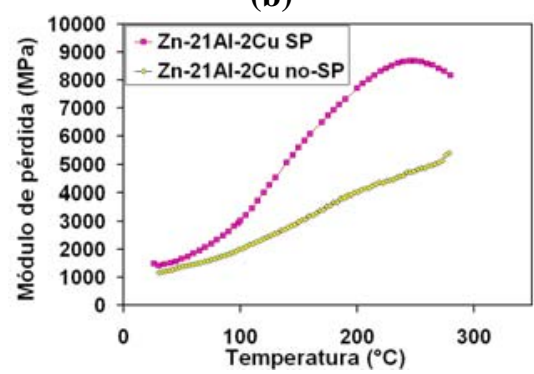

(d)

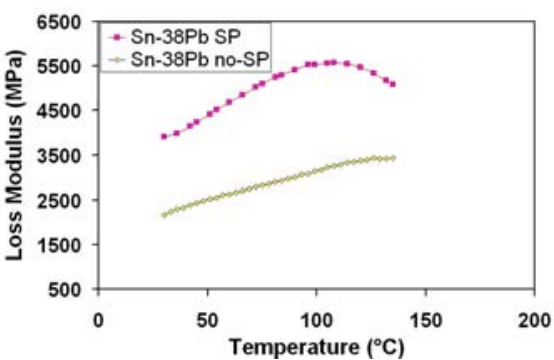

(f)

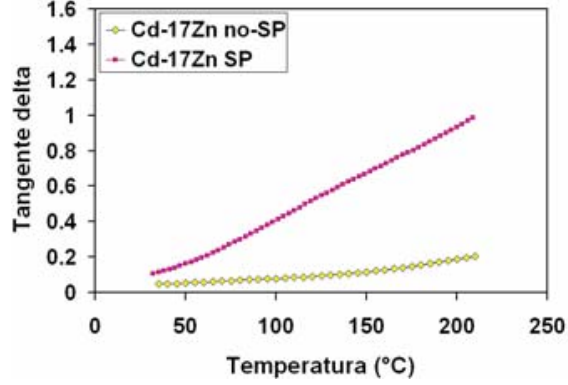

(h)

Figura 3: Variación del módulo de almacenamiento (a, b, c), el módulo de pérdida (d, e, f) y tan $\delta$ ( $g$, h, i) en función de la temperatura utilizando frecuencia de $1 \mathrm{~Hz}$, para las aleaciones $\mathrm{Zn}-21 \% \mathrm{Al}-2 \% \mathrm{Cu}$; $\mathrm{Cd}-17 \% \mathrm{Zn}$ y $\mathrm{Sn}-38 \% \mathrm{~Pb}$ laminadas (SP,- $\square-$ ) y en estado de colada (no-SP, $-\diamond_{-}$).

Las pruebas realizadas para la aleación $\mathrm{ZnAlCu}$ con microestructura de granos finos (superplásticas) se hicieron utilizando frecuencias entre 1 y $10 \mathrm{~Hz}$, ya que en las pruebas sobre la aleación de colada se 
observó que en este intervalo de frecuencias se tiene buena resolución, de los cambios en los valores de E', E" y $\tan \delta$, en tiempos de prueba cortos (aproximadamente 2 horas). Los resultados se presentan en la Figura $2(\mathrm{a}, \mathrm{b}, \mathrm{c})$. La respuesta viscoelástica del material se puede observar con buena resolución usando frecuencia de $1 \mathrm{~Hz}$.

El módulo de almacenamiento para el material laminado, Figura 2(a), también disminuye con la temperatura y la frecuencia, estos valores son menores que los obtenidos para la aleación en estado de colada, debido a que la muestra laminada tiene una microestructura de granos finos (superplástica, SP). Las curvas de módulo de pérdida, Figura 2(b), para la aleación en condición superplástica, presentan un comportamiento diferente al observado para la aleación en estado de colada, a la que llamaremos aleación en condición nosuperplástica, ver Figura 1(b). La aleación $\mathrm{ZnAlCu}$ con estructura superplástica presenta un máximo en las curvas de E" para cada una de las frecuencias evaluadas y toma valores diferentes en función de la frecuencia utilizada. Después de alcanzar este máximo el módulo de pérdida disminuye hasta alcanzar la temperatura de transformación eutectoide de la aleación.

Los valores de $\tan \delta$ para la aleación $\mathrm{ZnAlCu}$ superplástica, Figura 2(c), para todas las frecuencias evaluadas, llegan a ser aproximadamente tres veces más grandes que los observados para la aleación en condición no superplástica.

Los diversos fenómenos, deslizamiento de fronteras de grano, movimiento de átomos intersticiales o corrientes térmicas, que pueden dar origen a un pico en la curva de E" ocurren a una frecuencia determinada [12]. En nuestros experimentos con $\mathrm{ZnAlCu}$ superplástico ocurre un pico utilizando frecuencias entre 1 y 10 $\mathrm{Hz}$. Si este fenómeno es característico de la microestructura superplástica deberá repetirse en otros materiales superplásticos por lo que se realizaron pruebas en aleaciones $\mathrm{CdZn}$ y $\mathrm{SnPb}$ con estructura superplástica.

Los resultados obtenidos para cada una de estas aleaciones se presentan en la Figura 3(a - i). Para cada aleación se grafican las curvas de módulo de almacenamiento, tanto en condición superplástica como no-superplástica, ver Figura 3(a, b, c). Se puede observa que en todos los casos el módulo de almacenamiento disminuye monotónicamente con la temperatura. El de modulo de pérdida, Figura 3(d, e, f) presenta un máximo para cada una de las tres aleaciones en condición superplástica. Es importante hacer notar que ninguna de estas aleaciones en estado de colada presentó este máximo. Las temperaturas en las que se observa el máximo en la curva de módulo de pérdida son respectivamente: $108^{\circ} \mathrm{C}$ para $\mathrm{SnPb}, 137^{\circ} \mathrm{C}$ para $\mathrm{CdZn}$ y $250^{\circ} \mathrm{C}$ para $\mathrm{ZnAlCu}$. El módulo de pérdida es siempre mayor para las aleaciones en condición superplástica que para las aleaciones en estado de colada. Las curvas de $\tan \delta$, Figura $3(\mathrm{~g}, \mathrm{~h}, \mathrm{i})$, muestran que cuando las aleaciones en estudio tienen una microestructura de granos finos muestran un amortiguamiento mucho mayor que cuando estas aleaciones tienen la microestructura de colada.

\section{DISCUSION}

El máximo en la curva de módulo de pérdida ( $\left.\boldsymbol{E}^{\prime}\right)$ indica un tipo de transición cinética que sugiere un acomodamiento de la estructura de granos que antecede a la deformación superplástica, de la misma manera en que se puede observar la transición vítrea en materiales poliméricos. La palabra transición en este caso significa alteración de las propiedades macroscópicas del material al cruzar un umbral de temperatura.

En un material policristalino los granos vecinos pueden tener diferente orientación de sus bordes de grano con respecto a la dirección del esfuerzo aplicado, en consecuencia los bordes de grano que están bajo el efecto del máximo esfuerzo cortante serán los que se deslicen con mayor probabilidad de acuerdo con la ley de Schmid. Los bordes de grano que forman ángulos que no están en el intervalo de 35 a $45^{\circ}$, requerirán un mayor esfuerzo para resbalarse [14]. El esfuerzo requerido para el deslizamiento disminuirá con la temperatura, ya que a temperaturas más altas, mayor cantidad de bordes pueden resbalar incrementando el amortiguamiento del material, esto se debe a que se utilizará más energía para este deslizamiento entre bordes de grano. El estado superplástico se alcanzará cuando un máximo de bordes de grano esté listo para resbalarse.

La Figura 4 muestra el comportamiento del módulo de pérdida como función de la temperatura homóloga $\mathrm{T}_{\mathrm{H}}$, donde $\mathrm{T}_{\mathrm{H}}=\mathrm{T}_{\mathrm{abs}} / \mathrm{T}_{\mathrm{f}}\left(\mathrm{T}_{\mathrm{abs}}\right.$ es la temperatura de trabajo absoluta y $\mathrm{T}_{\mathrm{f}}$ es la temperatura de fusión). El máximo para cada curva está respectivamente en: 0.83 para la aleación $\mathrm{SnPb}, 0.69$ para la aleación $\mathrm{ZnAlCu}$ y 0.75 para la aleación $\mathrm{CdZn}$. Estos valores de $\mathrm{T}_{\mathrm{H}}$ coinciden con las temperaturas en las cuales se ha reportado el comportamiento superplástico de estas aleaciones $[\underline{9}, \underline{10}]$. 


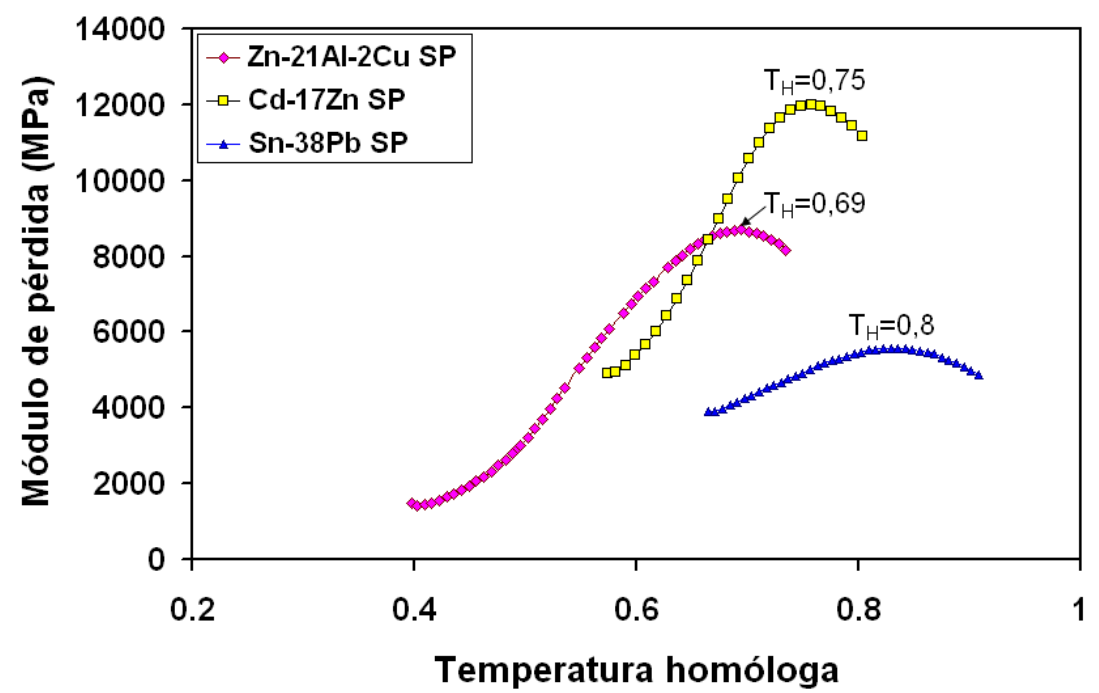

Figura 4: Módulo de pérdida en función de la temperatura homóloga para las aleaciones superplásticas Sn$38 \% \mathrm{~Pb}, \mathrm{Cd}-17 \% \mathrm{Zn}$ y $\mathrm{Zn}-21 \% \mathrm{Al}-2 \% \mathrm{Cu}$. Los máximos coinciden con la temperatura a la cual se obtiene la máxima superplasticidad observada.

NAZIRI et al [9] establecen que el comportamiento superplástico de la aleación ZnAlCu, depende fuertemente de la rapidez de deformación, el tamaño de grano y la temperatura de prueba ubicando este comportamiento en el intervalo de 200 a $250^{\circ} \mathrm{C}\left(0.63 \mathrm{~T}_{\mathrm{f}}-0.69 \mathrm{~T}_{\mathrm{f}}\right)$. Por otra parte AHMED y LANGDON [미] reportan que la aleación $\mathrm{Pb}-62 \% \mathrm{Sn}$ alcanza una superplasticidad excepcional a $140^{\circ} \mathrm{C}\left(0.90 \mathrm{~T}_{\mathrm{f}}\right)$. Durante el desarrollo de este trabajo se determino que la temperatura a la cual la aleación $\mathrm{Cd}-17 \% \mathrm{Zn}$ se comporta superplásticamente es $140^{\circ} \mathrm{C}\left(0.76 \mathrm{~T}_{\mathrm{f}}\right)$. Se puede observar que los valores de $\mathrm{T}_{\mathrm{H}}$ en el máximo de las curvas de $\boldsymbol{E}$ ” para cada aleación, corresponden con las temperaturas homólogas reportadas para el comportamiento superplástico de estas aleaciones. Considerando esta información, es posible que el incremento en el módulo de pérdida esté relacionado con el acomodamiento de los bordes de grano de un estado de no-deslizamiento a un estado de fácil deslizamiento. El acomodamiento de un gran número de bordes de grano se puede relacionar con el menor esfuerzo cortante requerido para superar la energía de cohesión entre los granos a la temperatura superplástica.

Los trabajos realizados hasta ahora [13-17] establecen que el deslizamiento de bordes de grano depende fuertemente del tipo de bordes y del ángulo de desorientación de los bordes. Cuando un policristal contiene diferentes tipos de bordes de grano, la magnitud de la contribución del deslizamiento puede variar de borde a borde y producir una deformación plástica heterogénea, provocando una fractura intergranular local. Los cambios requeridos en los bordes de grano para alcanzar un estado superplástico pueden ser los siguientes: como resultado de una fluctuación térmica de los átomos en cada grano hay suficiente energía para la difusión atómica en los bordes de grano en ausencia de fuerzas externas. Sin embargo, si se aplica una tensión uniaxial, la energía para la difusión puede ser menor en la dirección de máximo esfuerzo cortante, en consecuencia grupos de granos probablemente se moverán en conjunto a lo largo de bordes de grano cuya orientación sea muy próxima a la dirección de máximo corte, lo que dará origen a orientación preferencial de granos en relación con el eje de esfuerzo macroscópico. En la región plástica estas orientaciones preferenciales producirán bandas de deformación localizadas como las observadas por ASTANIN et al [18, 19]. El efecto del flujo de difusión disipará parte de la energía elástica almacenada en el sólido, lo cual en la escala macroscópica aparecerá como un máximo en la fricción interna del material también llamada tan $\delta$.

\section{CONCLUSIONES}

Usando DMA se pudo determinar la temperatura de transformación eutectoide de la aleación Zn$21 \% \mathrm{Al}$ modificada con $2 \% \mathrm{Cu}$.

Las tres aleaciones estudiadas, tanto en estado de colada como laminadas presentan un gran efecto viscoelástico a bajas frecuencias. Sin embargo, el amortiguamiento de las aleaciones en condición superplástica (granos finos) es considerablemente mayor que el de las aleaciones en estado de colada.

El análisis mecánico dinámico (DMA), permite estudiar la dependencia con la temperatura de la energía disipada durante cambios internos en el metal. Con este trabajo se mostró cómo el módulo de pérdida $\left(\boldsymbol{E}^{\prime \prime}\right)$ de estas aleaciones metálicas puede usarse para determinar la temperatura a la cual estas aleaciones metálicas se comportan superplásticamente. 
Es posible que el comportamiento superplástico sea una transición de orden superior, parecida a la transición vítrea observada en los polímeros, en donde el material acomoda su estructura de granos en la región elástica para que al atravesar el umbral de temperatura esté listo para la deformación superplástica.

\section{AGRADECIMIENTOS}

Los autores desean agradecer a Carmen Vázquez Ramos y Esteban Fregoso Israel quienes realizaron las mediciones en el equipo de análisis mecánico-dinámico.

\section{REFERENCIAS}

[1] MENARD, K.P., Dynamic Mechanical Analysis. A practical Introduction, 1 ed., USA, CRC Press, 1999.

[2] TURI, E.A. (Ed.), Thermal Characterization of Polymeric Materials, 2 ed., v. 1, New York, Academic Press, 1997.

[3] BUECHNER, P.M., STONE, D., LAKES, R.S., "Viscoelastic behavior of superplastic $37 \mathrm{wt} \% \mathrm{~Pb} 63$ wt $\%$ Sn over a wide range of frequency and time", Scripta Materialia, v. 41, n. 5, pp. 561-567, 1999.

[4] SÜLEYMAN GÜNDÜZ, "An internal friction study of a vanadium microalloyed steel by a dynamic mechanical thermal analyser", Turkish Journal of Engineering \& Environmental Sciences, v. 26, n. 4, pp. 353-359, 2002.

[5] VAN HUMBEECK, J., "Damping capacity of thermoelastic martensite in shape memory alloys", Journal of Alloys and Compounds, v. 355, n. 1-2, pp. 58-64, 2003.

[6] WANG, Q., FUSHENG, H., WU, J., GANGLING, H., "Damping behavior of porous CuAlMn shape memory alloy", Materials Letters, v. 61, n. 11-12, pp. 2598-2600, 2007.

[7] AALTIO, I., LAHELIN, M., SÖDERBERG, O., HECZKO, O., LÖFGREN, B., GE, Y., SEPPÄLÄ J., HANNULA, S.P., "Temperature dependence of the damping properties of Ni-Mn-Ga alloys", Materials Science and Engineering: A, v. 481-482, pp. 314-317, 2008.

[8] ZHANG, P., KONG, Q.P., ZHOU, H., "Dynamic internal-friction study of the superplastic deformation in a Pb-Sn alloy", Philosophical Magazine A, v. 77, n. 2, pp. 437-446, 1998.

[9] NAZIRI, H., PEARCE, R., "The influence of copper additions on the superplastic forming behaviour of the Zn-Al eutectoid", International Journal of Mechanical Science, v. 12, pp. 513-521, 1970.

[10] AHMED, M.M.I., LANGDON, T.G., "Exceptional ductility in the superplastic Pb- 62 Pct Sn eutectic", Metallurgical Transactions A, v. 8A, pp. 1832-1833, 1977.

[11] AMERICAN SOCIETY FOR METALS, METALS ABSTRACTS TRUST, METALS SOCIETY, Metals Abstracts Annual Index 1976, v. 9, Part B, American Society for Metals 1976.

[12] LAKES, R.S., Viscoelastic Solids, New York, CRC Press, pp. 1-9, 63-109,243-277, 1998.

[13] ZELIN, M.G., KRASILNIKOV, N.A., VALIEV, R.Z., GRABSKI, M.W, YANG, H.S., MUKHERJEE, A.K., " On the microstructural aspects of the nonhomogeneity of superplastic deformation at level of grain groups", Acta Metallurgica et Materialia, v. 42, n. 1, pp. 119-126, 1994.

[14] WATANABE, T., "Key issues of grain boundary engineering for superplasticity", Materials Science Forum 1997, v. 243-245, pp. 21-30, 1997.

[15] McNELLEY, T.R., McMAHON, M. E., "An investigation by interactive electron backscatter pattern analysis of processing and superplasticity in an aluminum-magnesium alloy", Metallurgical and Materials Transactions, v. 27A, n. 8, pp. 2252-2262, 1996. 
[16] PEREZ-PRADO, M.T., CRISTINA, M.C., TORRALBA, M., RUANO, O.A., GONZALEZ-DONCEL G., "Texture gradient evolution in Al-5\%Ca-5\%Zn sheet alloy after tensile deformation at high superplastic strain rate", Scripta Materialia, v. 35, n. 12, pp. 1455-1460, 1996.

[17] HSIAO, I.C., HUANG, J.C., "Characterization of grain boundary properties in superplastic al based alloys using EBSD”, Materials Science Forum, v. 357-359, pp. 381-386, 2001

[18] ASTANIN, V.V., KAIBYSHEV, O.A., FIZOVA, S.N., "Cooperative grain boundary sliding under superplastic flow”, Scripta Metallurgia et Materialia, v. 25, pp. 2663-2668, 1991.

[19] ASTANIN, V.V., KAIBYSHEV, O.A., FIZOVA, S.N., "The role of deformation localization in superplastic flow”, Acta Metallurgica et Materialia, v. 42, n. 8, pp. 2617-2622, 1994. 